\title{
Research Paper: Comparison of Selective Attention and Intelligence Profile in Bilingual and Monolingual Adolescents
}

\author{
*Rahim Yousefi' ${ }^{1}$, Mehran Soleymani ${ }^{1}$, Samira Ghazanfariyanpour ${ }^{1}$ \\ 1. Department of Psychology, Faculty of Psychology and Education, Azarbaijan Shahid Madani University, Tabriz, Iran.
}

\begin{tabular}{|c|c|}
\hline $\begin{array}{l}\text { Use your device to scan } \\
\text { and read the article online }\end{array}$ & Citation: Yousefi R, Soleymani M, Ghazanfariyanpour S. [Comparison of Selective Attention and Intelligence Profile in \\
\hline 口ifht: & $\begin{array}{l}\text { Bilingual and Monolingual Adolescents (Persian)]. Archives of Rehabilitation. 2018; 18(4):278-287. https://doi.org/10.21859/ } \\
\text { JREHAB.18.4.2 }\end{array}$ \\
\hline 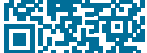 & d oil: https://doi.org/10.21859/JREHAB.18.4.2 \\
\hline
\end{tabular}

Received: 07 Jul. 2017 Accepted: 20 Nov. 2017

Keywords:

Attention, Intelli-

gence, Multilingual-

ism, Monolingual

\section{ABSTRACT}

Objective Cognitive abilities can be affected by bilingualism because of the close relationship between cognition and language. In the current study, selective attention and intelligence profile in adolescents who were dominant learner of English with those who were not learner of English were compared.

Materials \& Methods This study is a retrospective analysis and conducted by the cross-sectional method. This study included all adolescent who were dominant learners in English and non-learners adolescent (aged 13-15 years) in Isfahan in 2015. Adolescent aged 13-15 years $(n=64)$ in advanced levels of English (level RECE and REACH from the center of Iranian language and equivalent levels in other schools) and non-learner subjects (monolingual, $n=51$, control group) were selected from Region 3 of Isfahan. Adolescents in both groups were chosen from the same institute. Sampling was performed by non-random sampling method. To evaluate and measure adolescent's Intelligence profile in both groups, Gardner's multiple intelligence questionnaires were used. The classic Stroop test D-KEFS CW was used to measure the ability of selective attention of dominant learners in English and of non-learners adolescent. The data obtained by the classic Stroop test D-KEFS CW and Gardner's multiple intelligence questionnaires were analyzed through descriptive statistics and multivariate variance (MANOVA). Data were analyzed using SPSS version 22.

Results The results of the current study demonstrated that there is a significant difference between the two groups. Selective attention was different in dominant learners in English and non-learners adolescent as determined by the classic Stroop test D-KEFS CW $(P<0.05)$. This suggests that reaction time for learners was less than the non-learners adolescent in the classic Stroop task D-KEFS CW. The result indicates their faster performance and subsequently demonstrates that learner adolescent's functions are better than their non-learners counterparts in the classic Stroop test. Also, there is a significant difference between the scores of two groups in six components of intelligence profile including logicalmathematical intelligence and language intelligence $(P<0.001)$ as well as in interpersonal intelligence, musical intelligence, intrapersonal intelligence, and naturalist intelligence $(P<0.05)$. The results demonstrated that the scores of the learners were significantly higher than their peers' non-learners adolescent in the six components of multiple intelligences. This demonstrates that the learners were better than non-learners adolescent counterparts in the six components of the intellectual profile. The learners had better performances than their non-learners adolescent counterparts in selective attention, logicalmathematical intelligence, language intelligence, interpersonal intelligence, musical intelligence, intrapersonal intelligence, and naturalist intelligence.

Conclusion Learning a foreign language (e.g. English) may be an effective factor in selective attention and intelligence profile of adolescents. Therefore, the role of learning a foreign language should be considered in selective attention and intelligence profile of adolescents.

\section{* Corresponding Author:}

Rahim Yousefi, PhD

Address: Department of Psychology, Faculty of Psychology and Education, Azarbaijan Shahid Madani University, Tabriz, Iran.

Tel: +98 (914) 4612733

E-Mail: r.yousefi@gmail.com 


\title{
مقايسه توجه انتخابى و نيمرخ هوشى در نوجوانان دوزبانه و تكزبانه
}

\author{
"رحيم يوسفى'، مهران سليمانى'، سميرا غضنفريانيور'
}

ا - كروه روانشناسي، داتشكده علوم تربيتى وروانشناسى، دانشكاه شهيدمدنى آذربايجان، تبريز، ايران.

\begin{abstract}
حكبد

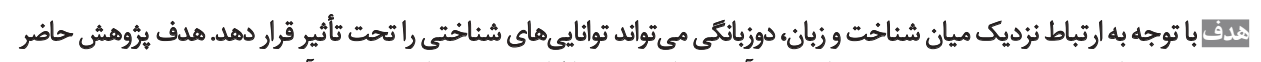

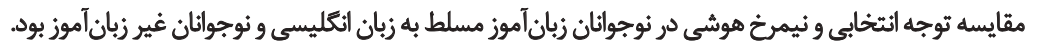

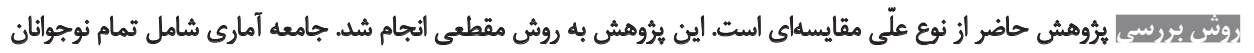

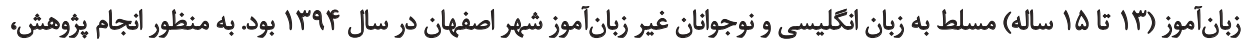

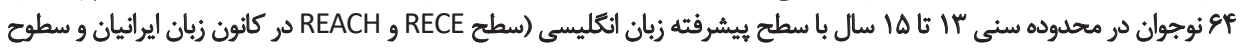

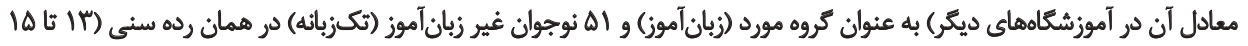

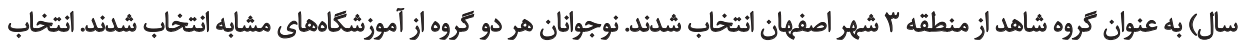

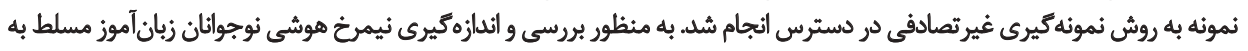

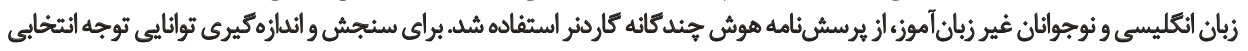

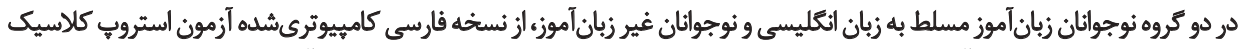

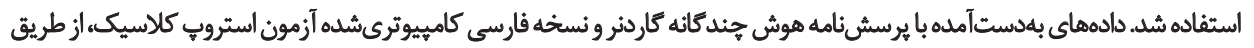

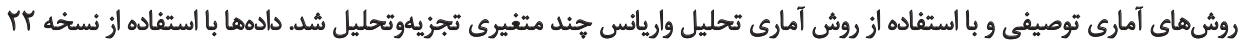

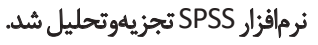

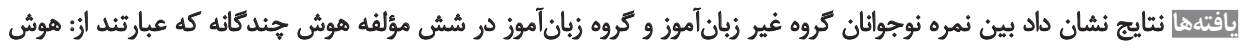

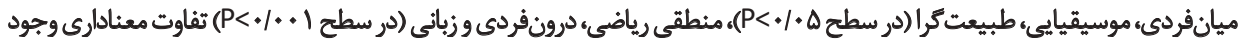

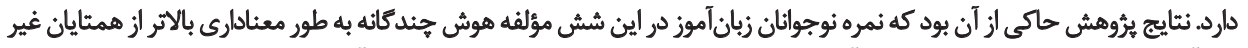

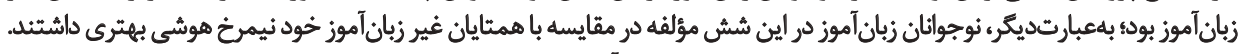

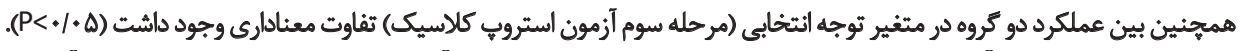

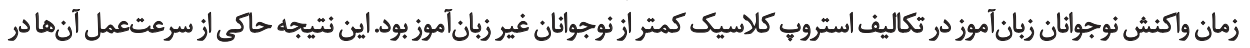

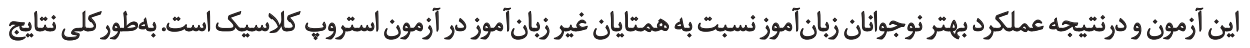

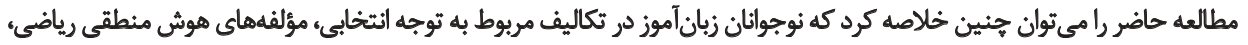

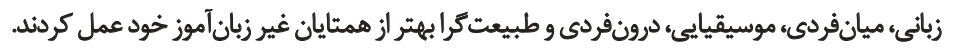

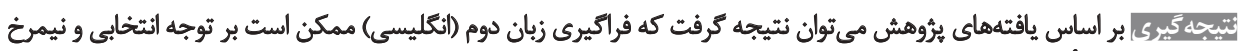

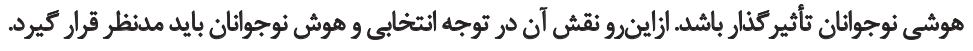

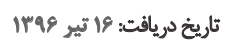

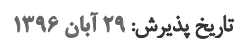

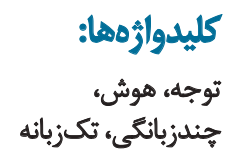

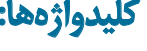

جندزبانكى، تكنزبانه

موقعيتهاى دنياى واقعى تعريف مى كند. از نظر بيكر [هاء دوز دوزبانه

مقدمه

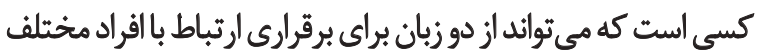

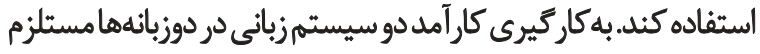

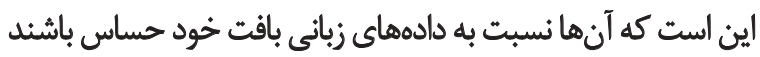

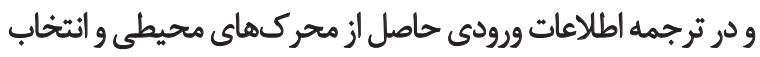

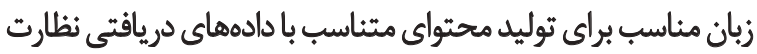

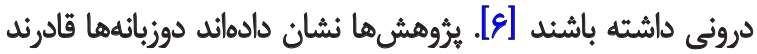

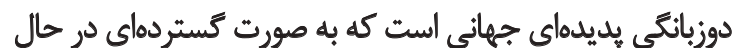

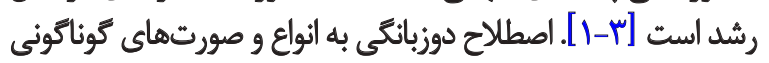

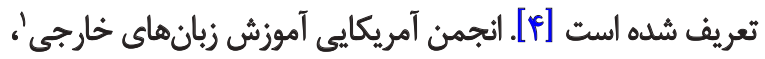
تسلط به زبان دوم را به صورت توانايى استفاده و به كاربرن زبان زبان در

1.American Council of Teaching Foreign Language (ACTFL)

-

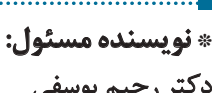

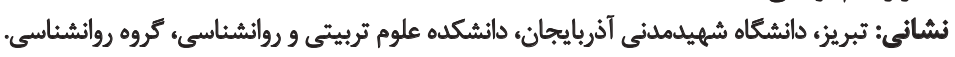

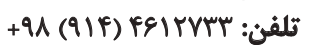
r.yousefi@gmail.com راياثامن: 
با محرك هاى مزاحم نياز دارد [ع] ]. اين مزاياى دوزبانكى در مطالعات

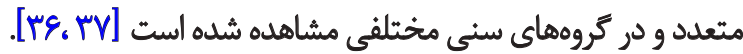

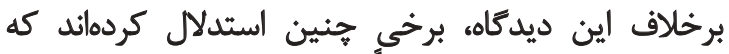

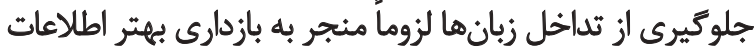

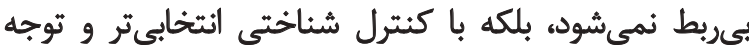

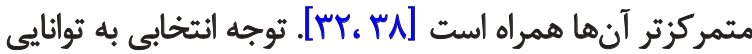

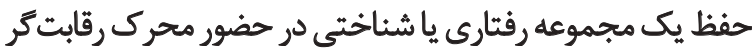

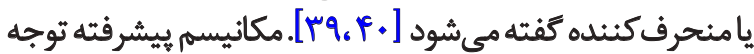

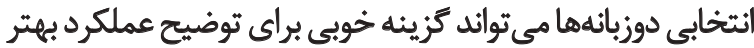
دوزبانهها در آزمونهائ هوشى نيز باشد.

انسان توانيىهاى ذهنى خود را از طريق تجربه كسب مي كئد.

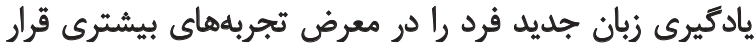

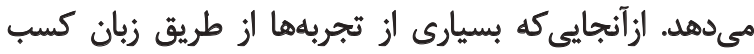

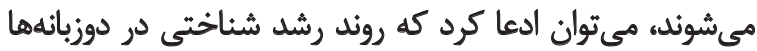

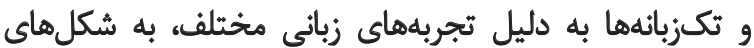

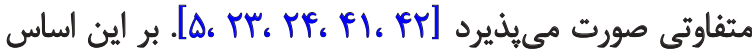

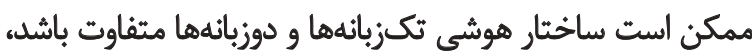

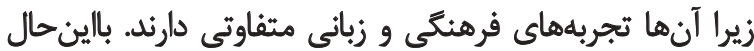

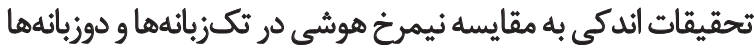

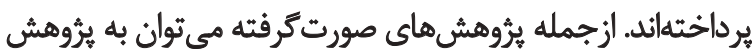

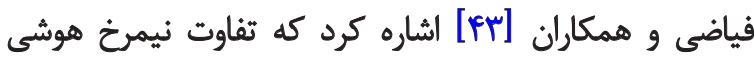

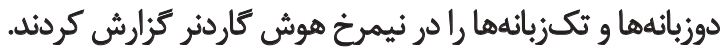

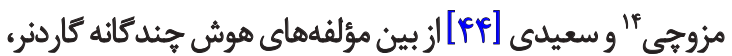

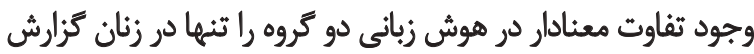

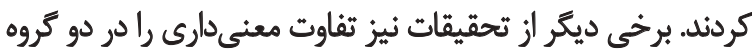

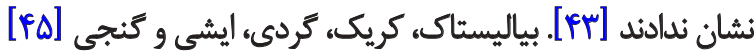

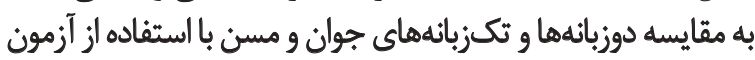

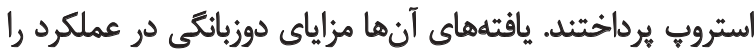

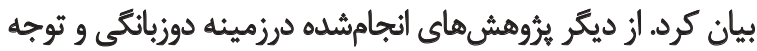

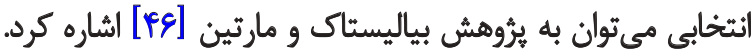

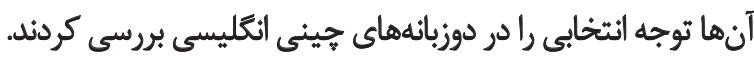

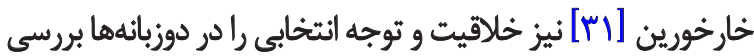

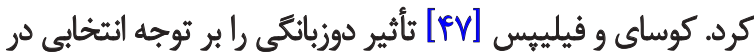
سالمندان بررسى كرد.

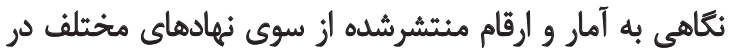

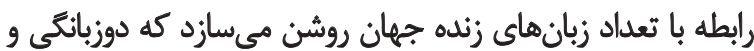

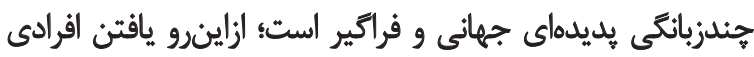

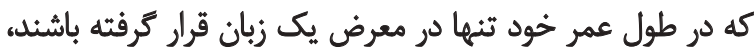

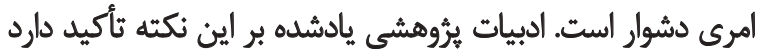

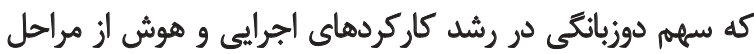

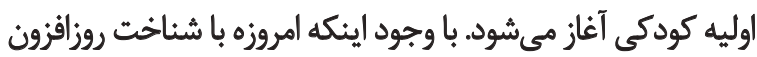

\section{Mazoochi}

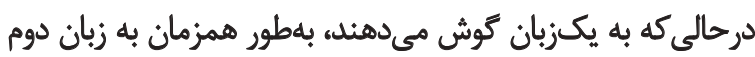

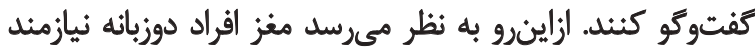

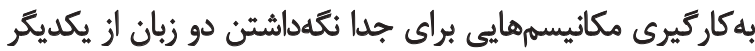

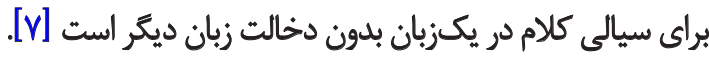
با توجه به جالش منحصربهفردى كه دوزيانهها براي مديريت دو

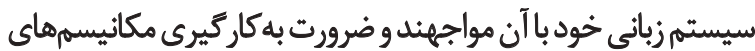

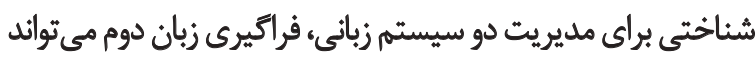

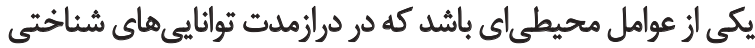

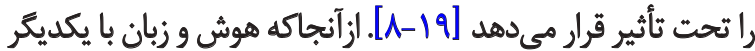

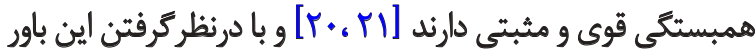

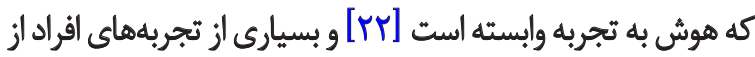

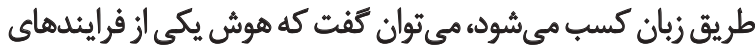

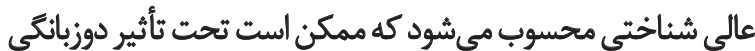

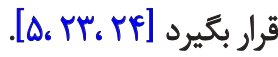

ديويد وكسلر هوش را نيرو يا ظرفيت كلى فرد براى رفتار و و

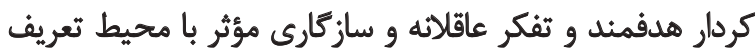

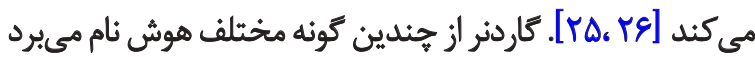

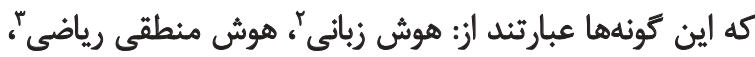

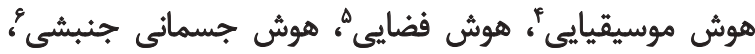

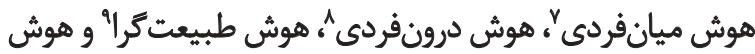

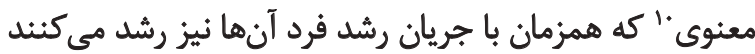

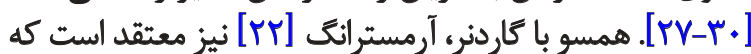

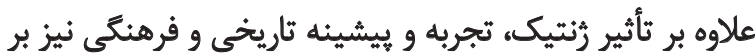

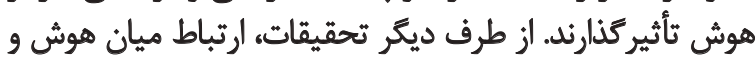

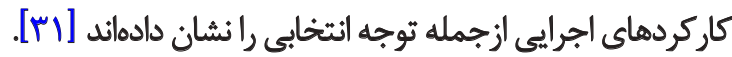

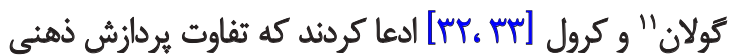

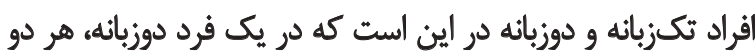

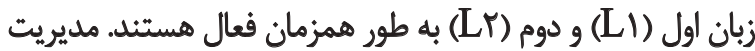

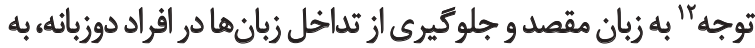

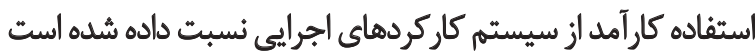

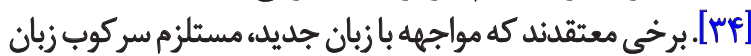

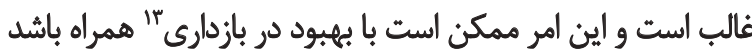

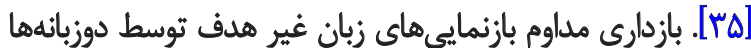

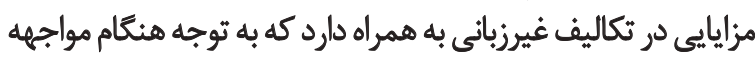

\section{Linguistic intelligence}

3. Logical-mathematical intelligence

4. Musical intelligence

5. Spatial intelligence

6. Bodily- kinethethic intelligence

7. Inter personal intelligence

8. Intra persona intelligence

9. Naturalist

10. Spiritual intelligence

11. Gollan

12. manage attention

13. Inhibition 
شركت در كلاسهاى آموزش زبان در مؤسسات آموزشى به مدت

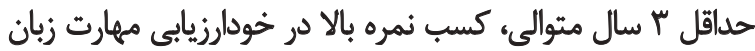

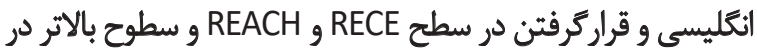

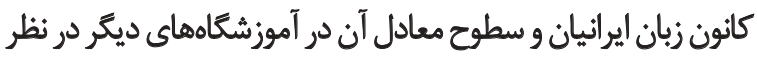

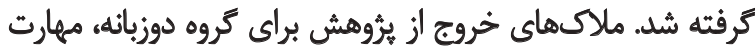

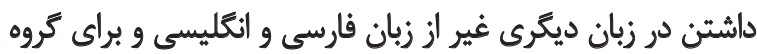

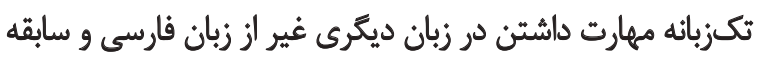

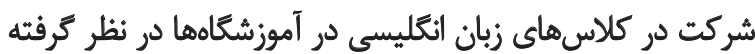

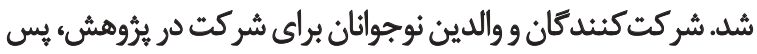

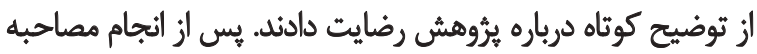

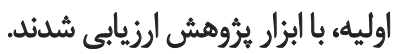

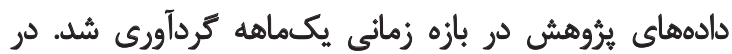

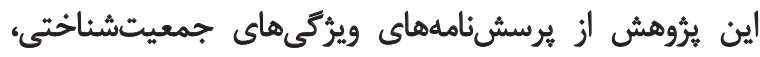

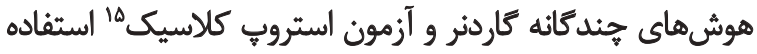

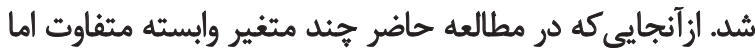

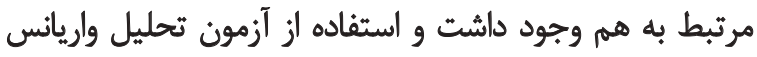

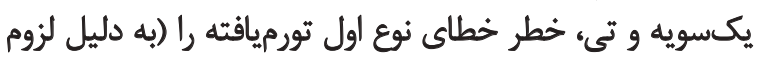

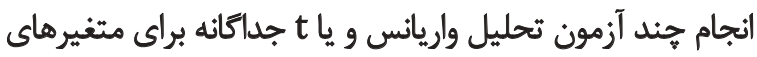

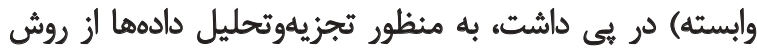

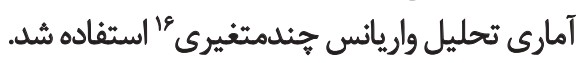

يرسش نامه /طلاعات جمعيتشُشناختي

در راستاى كنترل متغيرهاى فرهنتى و اقتصادى و انتخاب دقيق

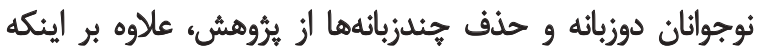

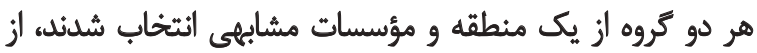

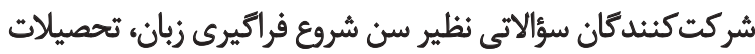

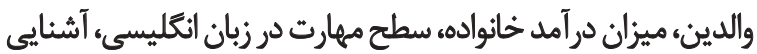

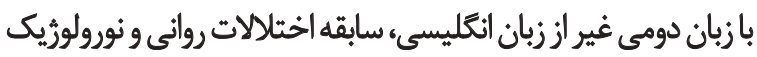
قبلى يا فعلى و سابقه آسيب به سر يرسيده شئد

$$
\text { أزمون استروب كلاسيك }
$$

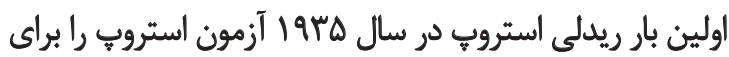

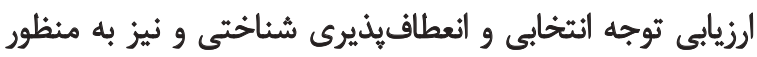

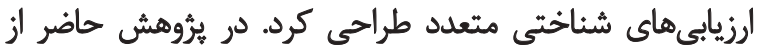

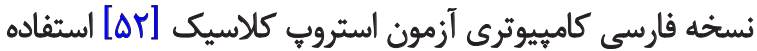

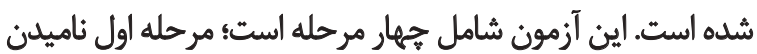

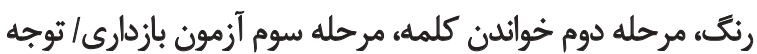

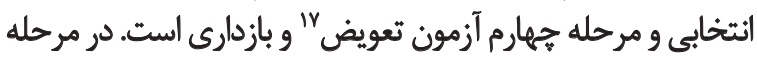

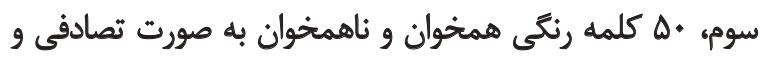

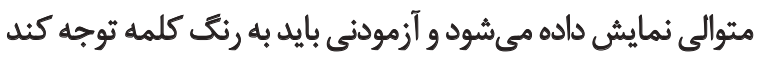

15. Delise Kaplan Executive Function System (D-KEFS) 16. MANOVA

17. Switching

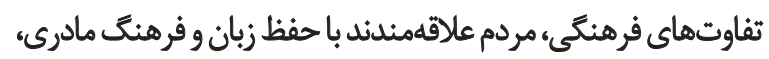

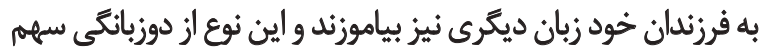

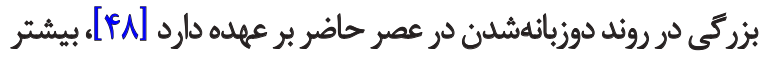

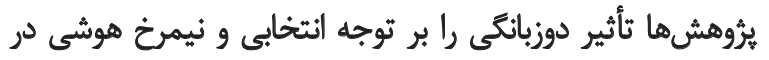

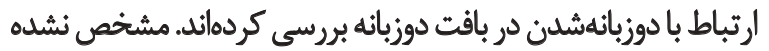

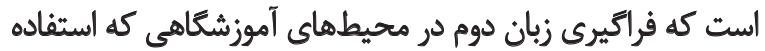

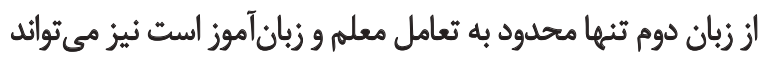
مزاياى يادشده را براى اين گروه از دوزبانهها دربرداشته باش باشد.

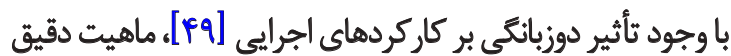

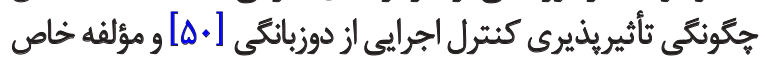

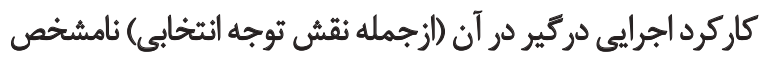

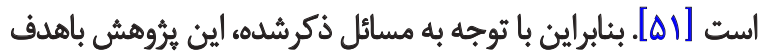

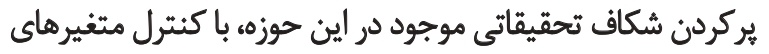

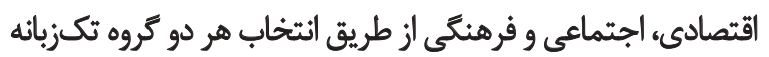

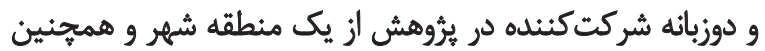

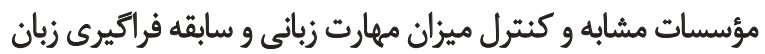

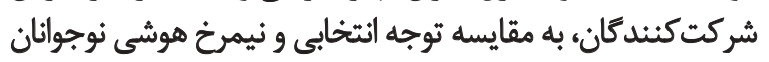

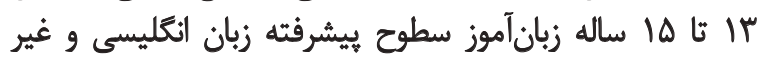
زبان آموز در شهر اصفهان يرداخته است.

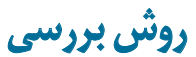

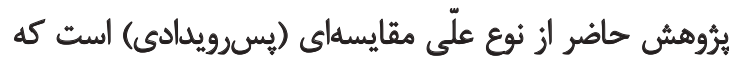

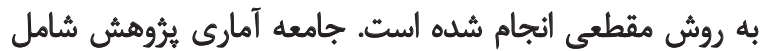

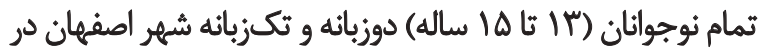

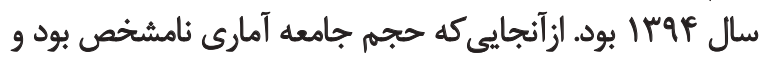

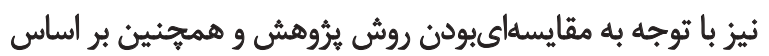

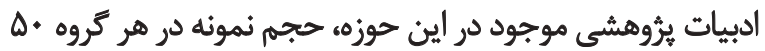

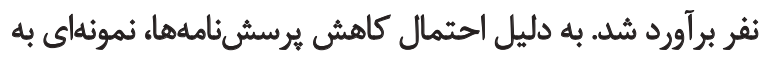

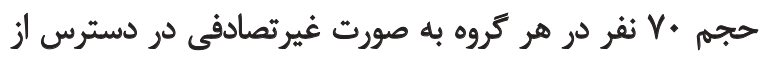

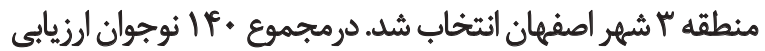

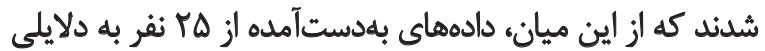

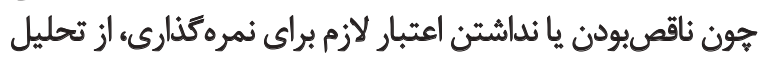

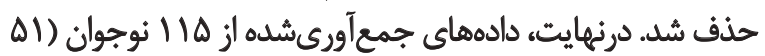

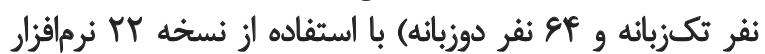

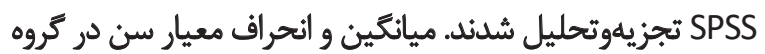

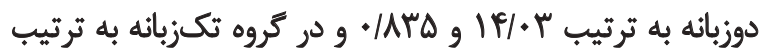

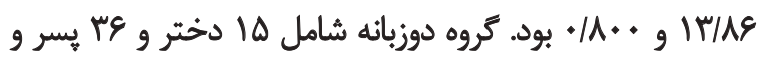

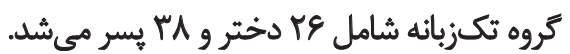
ملاكىهاى ورود به مطالعه براى كروه ثكزبانه، داشتن سلامت

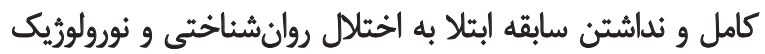

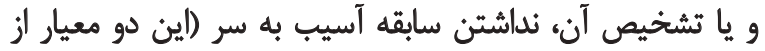

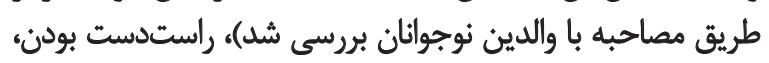

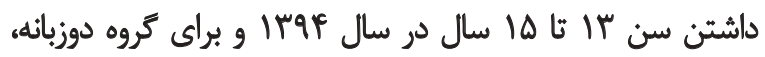


بررسى همكَنى واريانس متغيرها نشان داد مفروضه يكسانى باني

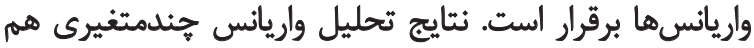
نشان داد اثثر اصلى شاخص ويلكز (P= (P)

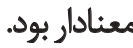

نتايج تحليل واريانس جندمتغيرى براي مقايسه دو كروه در توجه

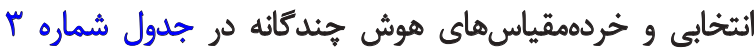

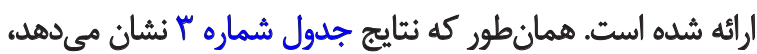

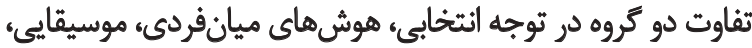

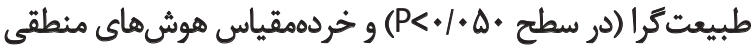

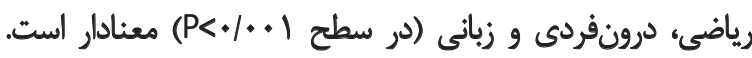

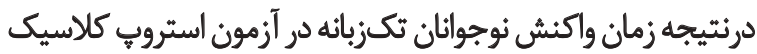

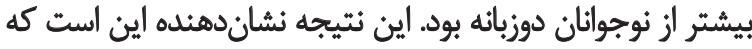

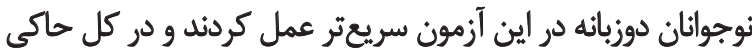

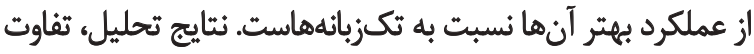

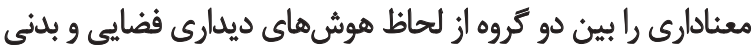

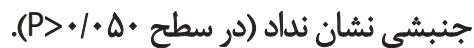

بحث

نتايج يُوهش حاضر نشان داد اثر استروب در نوجوائان تكزبانه

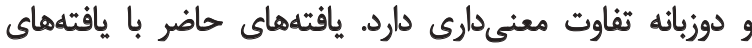

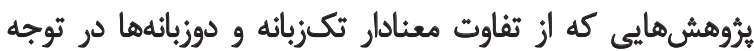

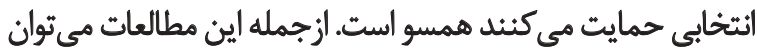

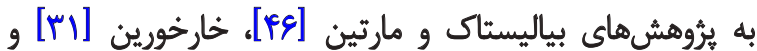

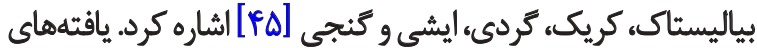

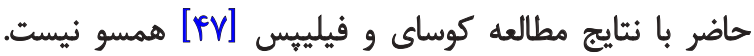

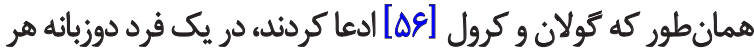

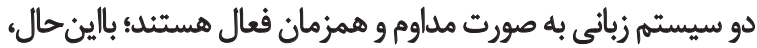

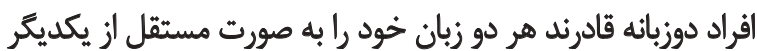

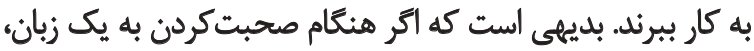

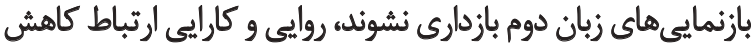

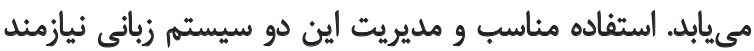

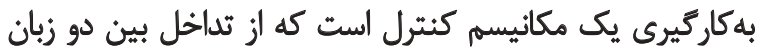

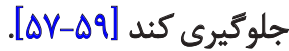

مؤلفه توجه كنترل اجرايى بلهشت در بازدارى موفق زبان

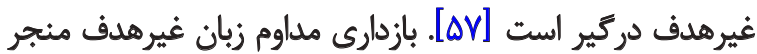

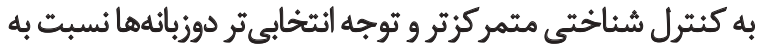

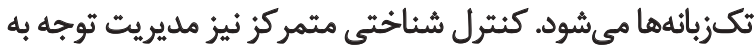

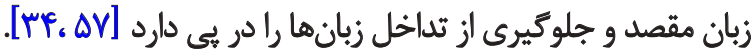

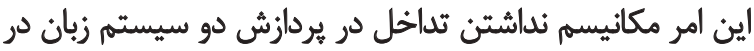

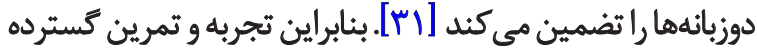

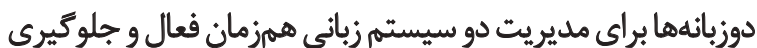

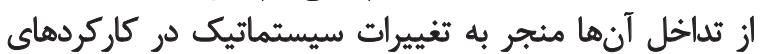

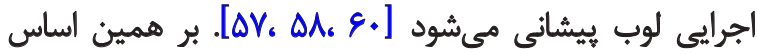

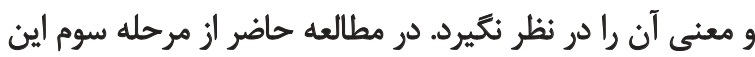

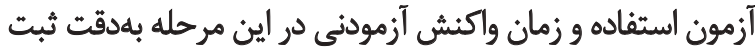

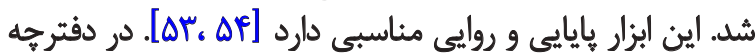

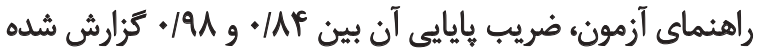

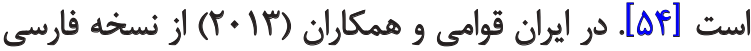

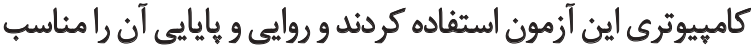

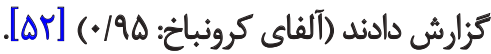

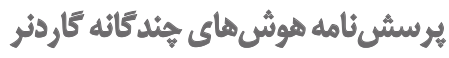

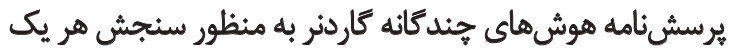

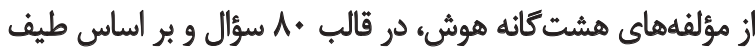

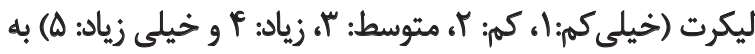

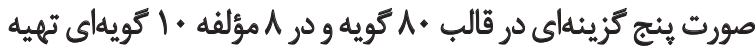

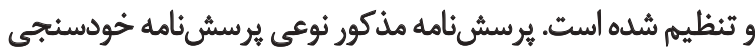

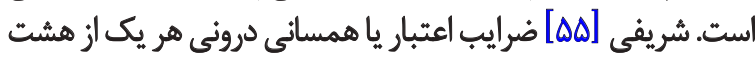

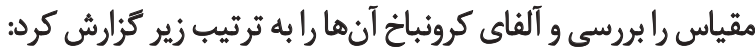

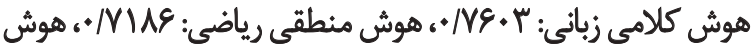

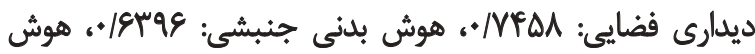

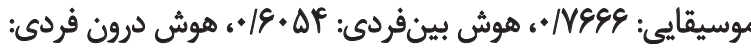

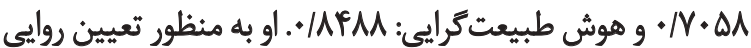

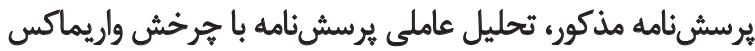

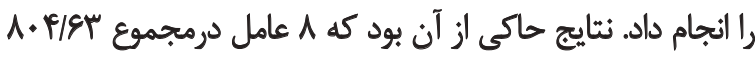

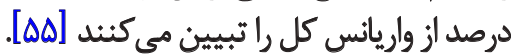

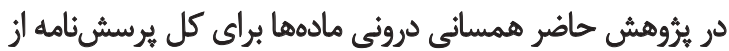

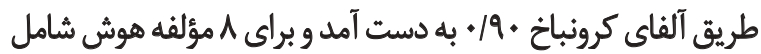

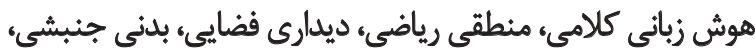

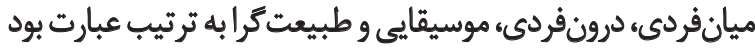

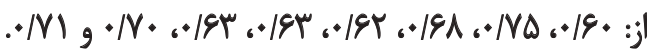

يافتهان

اطلاعات و يافتههاى جمعيتشناختى گروه نمونه مانند سن،

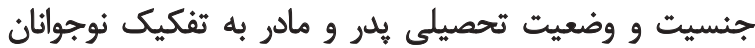

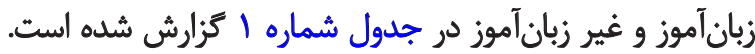

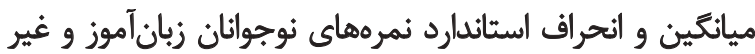

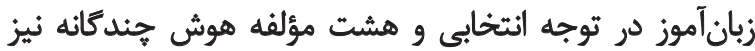

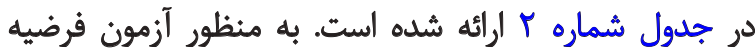

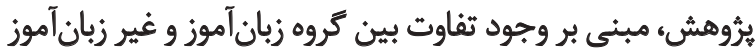

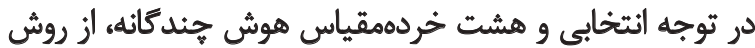

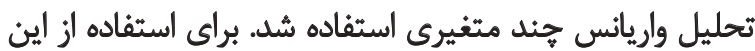

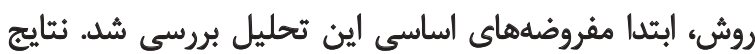

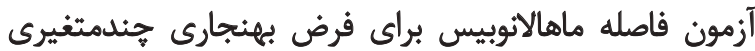
نشان داد در يثوهش هيج دادي جنادي يرت جيندمتغيرى وجود ندارد. نتايج حاصل أز آزمون لون براى هاي 
جدول ا. شاخصهاى جمعيت شُناختى زبان آموزان و كروه كنترل

\begin{tabular}{|c|c|c|c|c|}
\hline غيرزبان آموز (درصد) & زبان أموز (درصد) & \multicolumn{2}{|c|}{ سطح } & متغير \\
\hline I & if/.r & \multicolumn{2}{|c|}{ ميانكين } & \\
\hline$+/ \Lambda+$. & . ATts & \multicolumn{2}{|c|}{ اتحراف السيثاندارد } & سن ل سن \\
\hline $18($ (ับั) & $r E(P+1 \theta)$ & تعلداد (درصدر) & هي & \\
\hline$r E(V .18)$ & $r N(\Delta Q / P)$ & تعلداد (درصد) & 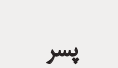 & جنسيت \\
\hline$\|(Y \backslash / E)$ & $r(g / \pi)$ & تعلداد (درصد) & زير دييلم & \\
\hline $\operatorname{lV}(\pi / \pi)$ & $r \cdot(r) / T)$ & تعلاد (درصد) & دييلم & \\
\hline$P(M / A)$ & $g(q / 4)$ & تعلداد (درصد) & فوقدييلم & \\
\hline $\operatorname{IV}(T / T)$ & $19(T \Delta)$ & تعلاد (درصد) & اليساتس & تحصيلات بلر \\
\hline$r(r / q)$ & $\operatorname{Ir}(\mathrm{NN})$ & تعلداد (درصد) & فوق ليسانس & \\
\hline$\cdot(*)$ & $g\left(q / r^{*}\right)$ & تعلداد (درصد) & دكترا & \\
\hline $\mid r(\pi / \Delta)$ & $P(g / \pi)$ & تعلداد (درصد) & زير دييلم & \\
\hline$r \cdot(Y Q T)$ & $M(M / A)$ & تعلداد (درصدي) & دييلم & \\
\hline$r(r / 9)$ & $\Delta(Y / A)$ & تعلاد (درصد) & فوقدييلم & \\
\hline $10($ (Y) & IN(YNI) & تعلداد (درصد) & ليسائس & تحصيلات مانر \\
\hline$r(r / q)$ & $I r(r+M)$ & تعلداد (درصد) & فوقليسانس & \\
\hline$\cdot(\cdot)$ & $r(f / V)$ & تعلداد (درصد) & دكترا & \\
\hline
\end{tabular}

فضايي بين دو كروه تفاوت معنادارى مشاهده نشد.در تبيين تفاوت

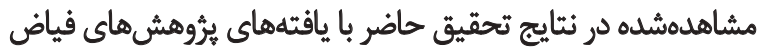

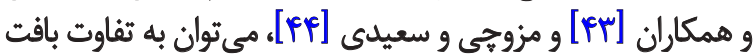

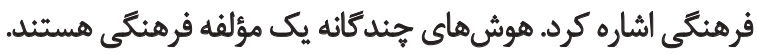

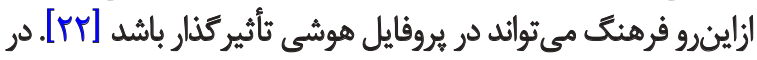

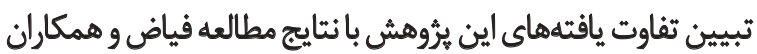

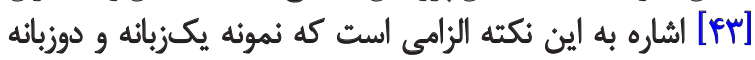
هيروهش حاضر از بافت فرهنكى يكسائى انتخاب شدند، درحالى كأله

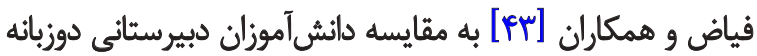

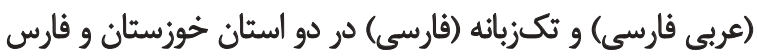

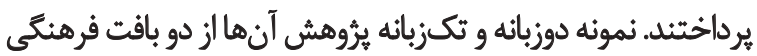
متفاوت اثتخاب شدند [FT.

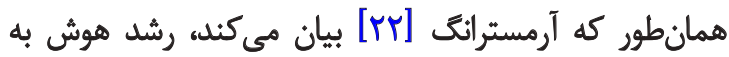

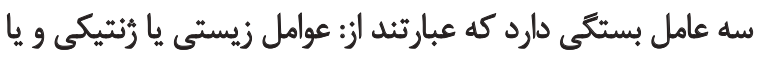

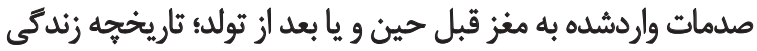

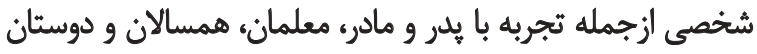

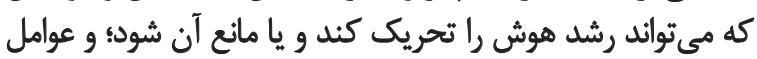

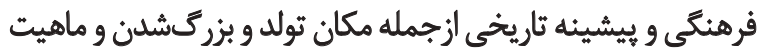

به نظر ميرسد نياز به كنترل تداخل كسترده بين دو زبان در

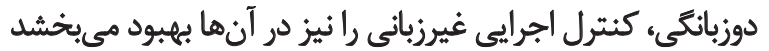

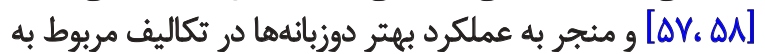

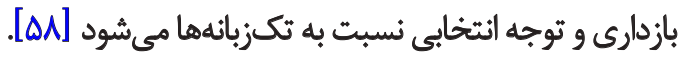
يافتههاي يثروهش حاضر نشان داد نمره دوزبانهها و تكىزبانهها

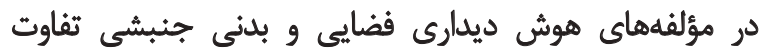

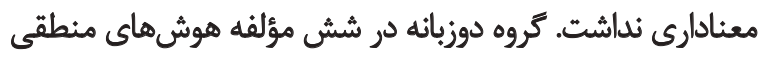

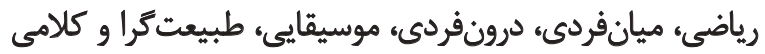

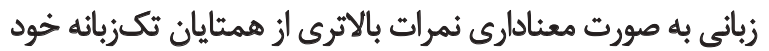

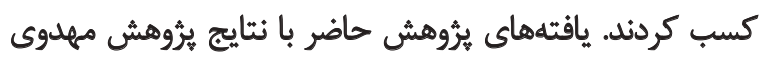

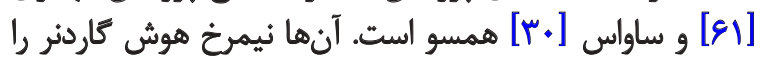

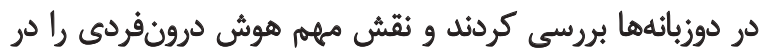

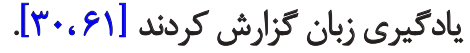

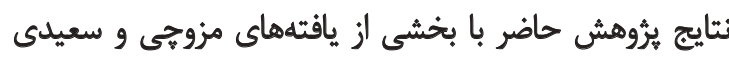

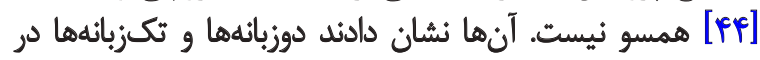

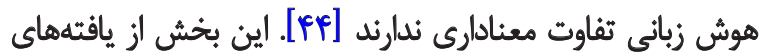

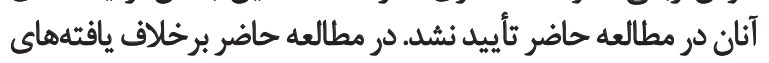

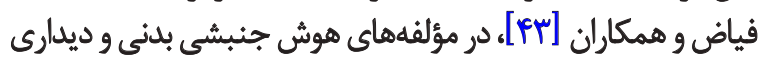




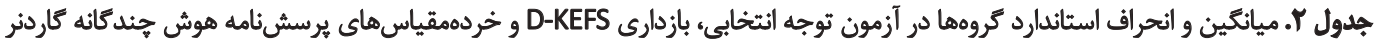

\begin{tabular}{|c|c|c|c|}
\hline انحراف استاندارد & مياتكين & 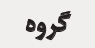 & حوزه \\
\hline r.Q/TAT & $9 v \cdot / 49 \%$ & دوزيانه & \multirow{3}{*}{ زمان واكثش در آزمون D-KEFS } \\
\hline & & & \\
\hline raV/NA & $11 E 9 / 49$ & تكريانه & \\
\hline$v / .$. & rV/VA & ل دوزبائه & \multirow{3}{*}{ هوش منطقى رياضى } \\
\hline & & & \\
\hline NEIA & $r / q$ & تكزبانه & \\
\hline $9 M .4$ & ralse & ل دوزياته & \multirow{3}{*}{ هوش ديلادى فضاييى } \\
\hline & & & \\
\hline$g / \Delta Q$. & $\mathrm{ra/u}$ & تكزبانه & \\
\hline$\Delta / \mp \notin \varnothing$ & relse & ا دوزباته & \multirow[b]{2}{*}{ هوش بدنى جنبشى } \\
\hline$\Delta / r+\Delta$ & $r V / s q$ & تكنزبانه & \\
\hline s/qTा & $r V / \wedge)$ & 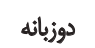 & \multirow{3}{*}{ هوش ميانفردى } \\
\hline & & & \\
\hline$\Delta / N \cdot Y$ & Me/Fa & تكنزيائه & \\
\hline G/DPA & MIAT & 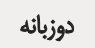 & \multirow{3}{*}{ هوش درونفردى } \\
\hline & & & \\
\hline$V / \Delta I I$ & $\pi / \Delta \omega$ & تكرزيانه & \\
\hline$Q \cdot A r$ & $r e / q^{2}$ & دوزبائه & \multirow{3}{*}{ هوش موسيقايع } \\
\hline & & & \\
\hline NATE & $\pi / 99$ & تكزيانه & \\
\hline$V / r+q$ & $r \Delta / \Delta$ & ل دوزباته & \multirow{3}{*}{ هوش طبيعت را } \\
\hline & & & \\
\hline$\Delta / A r I$ & ry/Ne & تكنزبانه & \\
\hline$g / A+Y$ & $r e / r r$ & دوزياته & \multirow[b]{2}{*}{ هوش زيانى } \\
\hline NGqF & זq/ ו & تكنيائه & \\
\hline
\end{tabular}

توانبخننى

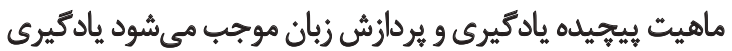

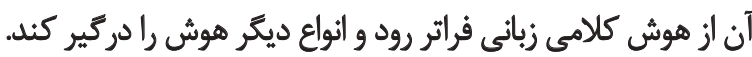

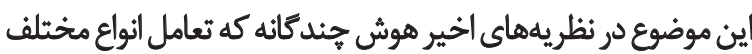

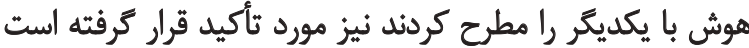

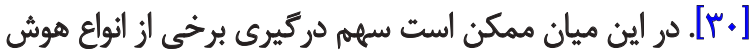

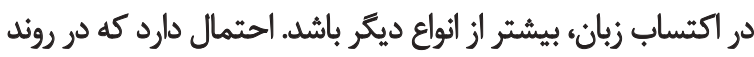

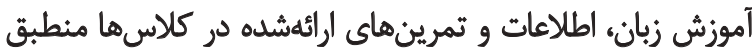

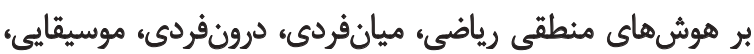

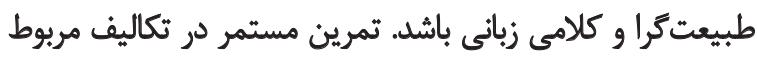

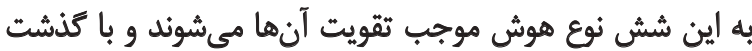

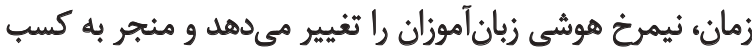

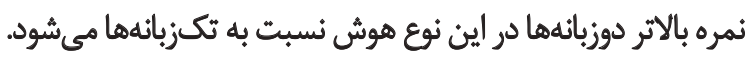

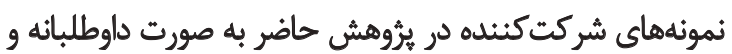

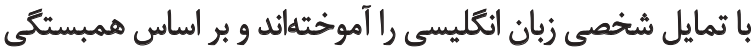

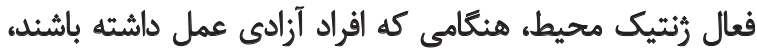

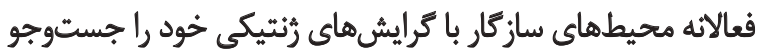

تحولات فرهنگى و تاريخى در حوزهاى مختلف. بنابراين تكامل

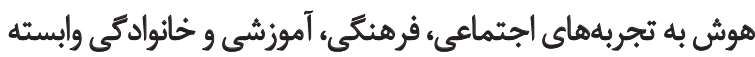

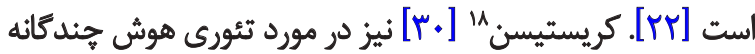

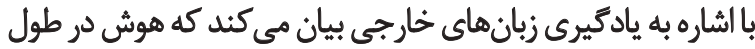

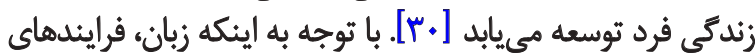

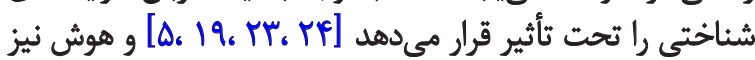

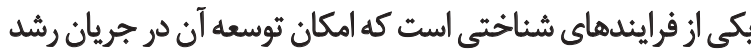

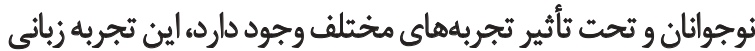

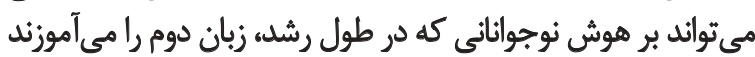

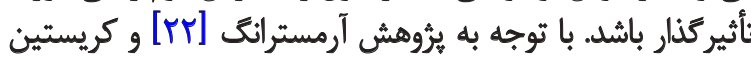

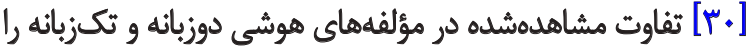

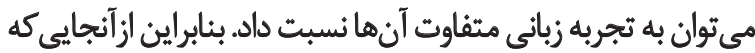

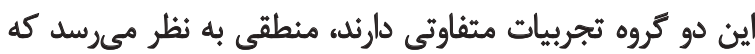
نيمرخهاى هوشى متفاوتى نيز داشته باشند.

\section{Christison}




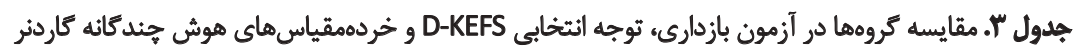

\begin{tabular}{|c|c|c|c|c|c|c|c|}
\hline توان آمارى & اندازه اثر & sig. & $\mathbf{F}$ & ميانكين مجذورات & درجه آزادى & مجموع مجذورات & مثغير وابسته \\
\hline . IAVA & $1 \circ A^{\circ}$ & $\% .+r$ & VAPQ & 1.ATPAY/ATA & 1 & 1.ATPAY/ATA & زمان واكنش در أزمون D-KEFS \\
\hline ./9ve & ( &.. .1 & $10 / M A$ & $9 Q 1 / M F F$ & 1 & $901 / \pi P F$ & هوش هنطقى رياضى \\
\hline .1 .01 & $.1 .+1$ & - NAS & $+1 \cdot Y a$ & r/ur & 1 & t/uAT & هوش ديدارى فضايع \\
\hline.$/ N r$ & .10 .9 & $.4 m$ & $V \cdot M$ & $r+/ 11 r$ & 1 & $r+/ 1 \%$ & هوش بدنى جنبشى \\
\hline$\cdot M+1$ & $.1 \cdot \Delta A$ & $\%$ & $8 / 911$ & TAF/MQP & 1 & YAF/TRE & هوش هيان فردى \\
\hline.$/ 94$. &.$M M$ & $.1 . .1$ & $|\Delta|+\not F \mid$ & $n+\Delta / A \cdot r$ & 1 & $n+\Delta / \lambda+r$ & هوش درونفردى \\
\hline $.18+$. &.$/ .4 r$ & $\%$ \%A & $r / u r$ & rAQ/PrT & 1 & rAVART & هوش موسيقايي \\
\hline . /ATA & $\% n r$ & $\%$ & NATY & rMA.\& & 1 & TMNO. & هوش طبيعتكرا \\
\hline.$/ 94$. & .1 .94 & $1 . .01$ & W/AM & EATIAEA & 1 & EAT/AEA & هوش زيانى \\
\hline
\end{tabular}

در سطوح RECE , REACH، ملاك دوزبائكي بود و مهارت زباني

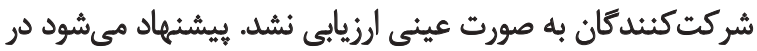

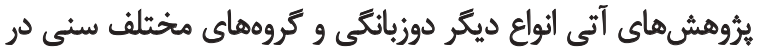

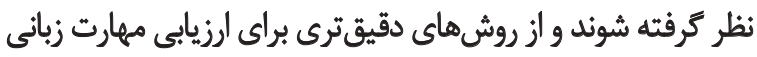

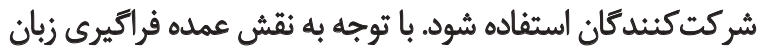

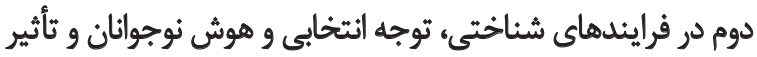

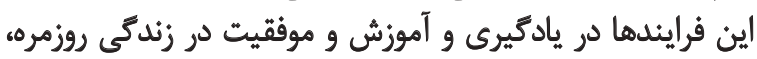

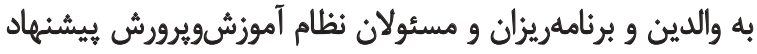

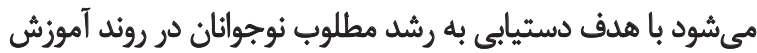

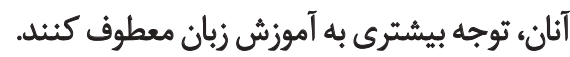

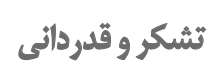

اين مقاله از يايانثامه مقطع كارشناسى ارشد خانم سميرا

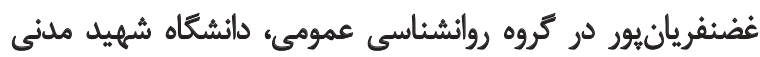

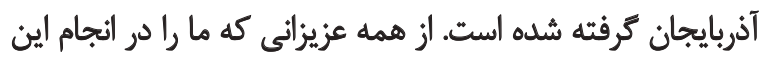

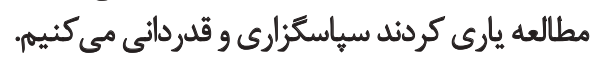

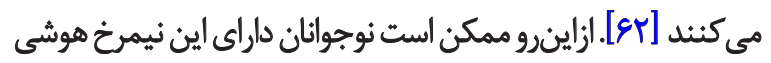

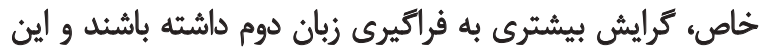

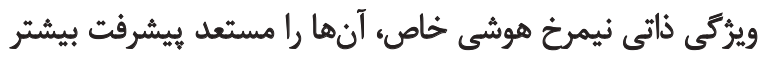

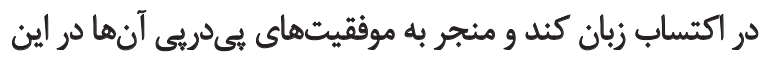

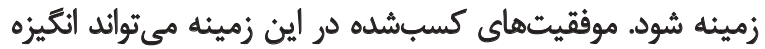

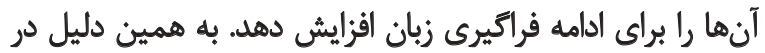

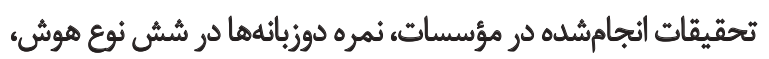

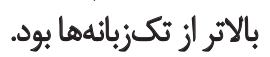

\section{S. Sdr}

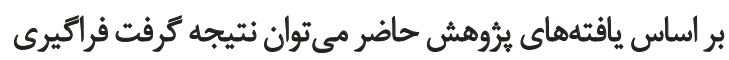

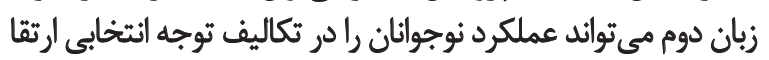

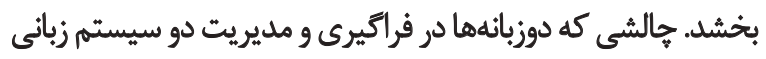

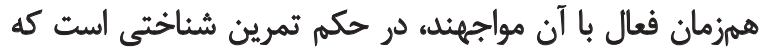

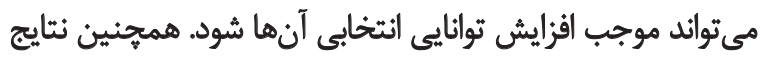

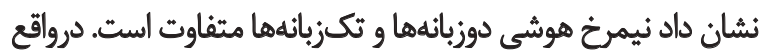

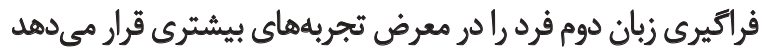

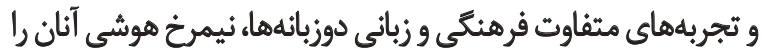

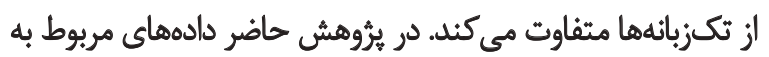

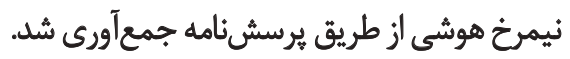

از محدوديتهاى يثزوهش حاضر مىتوان به جمع آورى دادهها از

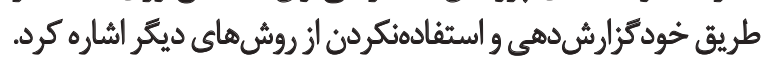

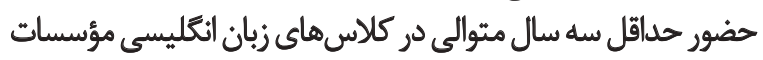

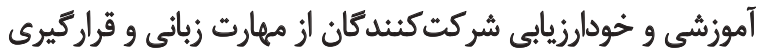




\section{References}

[1] Bhatia TK, Ritchie WC. The handbook of bilingualism. New Jersey: John Wiley \& Sons; 2008.

[2] Bhatia TK, Ritchie WC. Bilingualism and multilingualism in the global media and advertising. The Handbook of Bilingualism and Multilingualism. 2012; 563-97. doi: 10.1002/9781118332382.ch23

[3] Mohammadi H, Yadegari F, Nili Pour R, Rahgozar M. [Prevalence of stuttering in Javanroud's bilingual students (Persian)]. Archives of Rehabilitation. 2008; 9(1):43-8.

[4] Mohammadi H, Nili Pour R, Yadegari F, Karimlou M. [Comparison of linguistic knowledge between students and their healthy peers (Persian)]. Archives of Rehabilitation. 2008; 9(2):47-51.

[5] Baker C. Foundations of bilingual education and bilingualism. Tonawanda: Multilingual Matters; 2011.

[6] Green DW. Mental control of the bilingual lexico semantic system. Bilingualism: Language and Cognition. 1998; 1(2):67. doi: 10.1017/ s1366728998000133

[7] Ibrahim R. Literacy problems in Arabic: Sensitivity to diglossia in tasks involving working memory. Journal of Neurolinguistics. 2011; 24(5):571-82. doi: 10.1016/j.jneuroling.2010.10.003

[8] Ben Zeev S. Mechanisms by which childhood bilingualism affects understanding of language and cognitive structures. Bilingualism: Psychological, social, and educational implications. 1977:29-55.

[9] Hakuta K, Diaz RM. The relationship between degree of bilingualism and cognitive ability: A critical discussion and some new longitudinal data. Children's Language. 1985; 5:319-44.

[10] Kessler C, Quinn ME. Language minority children's linguistic and cognitive creativity. Journal of Multilingual \& Multicultural Development. 1987; 8(1-2):173-86. doi: 10.1080/01434632.1987.9994284

[11] Bialystok E. Bilingualism in development: Language, literacy, and cognition. Cambridge: Cambridge University Press; 2001.

[12] Romaine S. Bilingualism. New Jersey: Wiley Blackwell; 1995.

[13] Polk TA, Farah MJ. The neural development and organization of letter recognition: Evidence from functional neuroimaging, computational modeling, and behavioral studies. The National Academy of Sciences; 1998; 95(3):847-52. doi: 10.1073/pnas.95.3.847

[14] Salthouse TA, Mitchell DR. Effects of age and naturally occurring experience on spatial visualization performance. Developmental Psychology. 1990; 26(5):845-54. doi: 10.1037/0012-1649.26.5.845

[15] Maguire EA, Gadian DG, Johnsrude IS, Good CD, Ashburner J, Frackowiak RSJ, et al. Navigation related structural change in the hippocampi of taxi drivers. The National Academy of Sciences; 2000; 97(8):4398-403. doi: 10.1073/pnas.070039597

[16] Green CS, Bavelier D. Action video game modifies visual selective attention. Nature. 2003; 423(6939):534-7. Doi: 10.1038/ nature 01647

[17] Martin Rhee MM, Bialystok E. The development of two types of inhibitory control in monolingual and bilingual children. Bilingualism: Language and Cognition. 2008; 11(01). doi: 10.1017/ s1366728907003227
[18] Paradis J, Nicoladis E, Genesee F. Early emergence of structura constraints on code-mixing: Evidence from French-English bilingual children. Bilingualism: Language and Cognition. 2000; 3(3):24561. doi: $10.1017 /$ s1366728900000365

[19] Bialystok E, Craik FIM. Cognitive and linguistic processing in the bilingual mind. Current Directions in Psychological Science. 2010; 19(1):19-23. doi: 10.1177/0963721409358571

[20] Oller JW, Perkins K. A further comment on language proficiency as a source of variance in certain affective measures. Language Learning. 1978; 28(2):417-23. doi: 10.1111/j.1467-1770.1978.tb00144.x

[21] Genesee F. The role of intelligence in second language learning1. Language Learning. 1976; 26(2):267-80. doi: 10.1111/j.14671770.1976.tb00277.x

[22] Armstrong T. The multiple intelligences of reading and writing: Making the words come alive. Virginia: ASCD; 2003.

[23] Cummins J. Cognitive/academic language proficiency, linguistic in terdependence, the optimum age question and some other matters. Working Papers on Bilingualism Toronto. 1979; 19:197-202.

[24] Shapson S, D'Oyley V. Bilingual and multicultural education: Canadian perspectives. Bristol: Multilingual matters; 1984.

[25] Phares J, Teral T. Clinical phsychiligy: Concepts methods and profcasion [M. Firooz Bakht, Persian Trans). Tehran: Roshd; 2010.

[26] Mahmoudi Raad M, Arasteh HR, Afghah S, Barati Sadeh F. [The role of communication skills and social problem solving training on self esteem and IQ in third grade students (Persian)]. Archives of Rehabilitation. 2007; 8:71-6.

[27] Rouhshad A. Second language attrition and activation: Are multiple intelligence profiles and semantic categorization playing a role. Porta Linguarum. 2011; 15:135-49

[28] Badiee E, Farajollahi M. The impact of E-Content based on gard ner's intrapersonal and interpersonal intelligences on students learning. ICT in Education. 2014; 4(16):23-38.

[29] Vaezi S, Zolfaghari AF, Rahimi E. Cognitive processing in billngual and monolingual children. Thinking and Children. 2012; 3(1):119134.

[30] Savas P. Pre-service English as a foreign language teachers' perceptions of the relationship between multiple intelligences and foreign language learning. Learning and Individual Differences. 2012; 22(6):850-5. doi: 10.1016/j.lindif.2012.05.003

[31] Kharkhurin AV. The role of selective attention in bilingual creativity. Creativity Research Journal. 2011; 23(3):239-54. doi: 10.1080/10400419.2011.595979

[32] Hommel B, Colzato LS, Fischer R, Christoffels IK. Bilingualism and creativity: Benefits in convergent thinking come with losses in divergent thinking. Frontiers in Psychology. 2011; 2. doi: 10.3389/ fpsyg.2011.00273

[33] Paradis M. The cognitive neuropsychology of bilingualism. In: de Groot AMB, Kroll JF, editors. Tutorials in bilingualism: Psycholin guistic perspectives. Mahwah: Lawrence Erlbaum Associates; 1997.

[34] Morales J, Calvo A, Bialystok E. Working memory development in monolingual and bilingual children. Journal of Experimental Child Psychology. 2013; 114(2):187-202. doi: 10.1016/j.jecp.2012.09.002 
[35] Abutalebi J, Green DW. Control mechanisms in bilingual language production: Neural evidence from language switching studies. Language and Cognitive Processes. 2008; 23(4):557-82. doi: 10.1080/01690960801920602

[36] Bialystok E, Craik FIM, Ryan J. Executive control in a modified antisaccade task: Effects of aging and bilingualism. Journal of Experimental Psychology: Learning, Memory, and Cognition. 2006; 32(6):1341-54. doi: 10.1037/0278-7393.32.6.1341

[37] Bialystok E, Craik FIM, Klein R, Viswanathan M. Bilingualism, aging, and cognitive control: Evidence from the simon task. Psychology and Aging. 2004; 19(2):290-303. doi: 10.1037/0882-7974.19.2.290

[38] Colzato LS, Bajo MT, van den Wildenberg W, Paolieri D, Nieuwenhuis S, La Heij W, et al. How does bilingualism improve executive control? A comparison of active and reactive inhibition mechanisms. Journal of Experimental Psychology: Learning, Memory, and Cognition. 2008; 34(2):302-12. doi: 10.1037/0278-7393.34.2.302

[39] Joseph JS, Chun MM, Nakayama K. Attentional requirements in a "preattentive" feature search task. Nature. 1997; 387(6635):805-7. doi: $10.1038 / 42940$

[40] Neumann E, DeSchepper BG. Costs and benefits of target activation and distractor inhibition in selective attention. Journal of Experimental Psychology: Learning, Memory, and Cognition. 1991; 17(6):1136-45. doi: 10.1037/0278-7393.17.6.1136

[41] Peal E, Lambert WE. The relation of bilingualism to intelligence. Psychological Monographs: General and Applied. 1962; 76(27):1-23. doi: $10.1037 /$ h0093840

[42] Naseri E, Nejad Ansari D. The relationship between multiple intelligences and Iranian high school students' L2 writing achievement. International Journal of Psychology and Behavioral Research. 2013; 2(5):282-90.

[43] Fayyazi A, Sahragard R, Roshan B, Zandi B. Bilingual and monolingual differences on self-estimates of mutiple intellgences regerding gender: A study of high school students in iran. International Journal of Language Learning and Applied Linguistics World. 2013; 4(4):513-33.

[44] Saeidi M, Mazoochi N. A comparative study on bilingual and monolingual Iranian EFL learners' linguistic intelligence across genders. Life Science Journal. 2013; 10:321-34.

[45] Bialystok E, Craik FIM, Grady C, Chau W, Ishii R, Gunji A, et al. Effect of bilingualism on cognitive control in the Simon task: Evidence from MEG. NeuroImage. 2005; 24(1):40-9. doi: 10.1016/j. neuroimage.2004.09.044

[46] Bialystok E, Martin MM. Attention and inhibition in bilingual children: Evidence from the dimensional change card sort task. Developmental Science. 2004; 7(3):325-39. doi: 10.1111/j.14677687.2004.00351.x

[47] Kousaie S, Phillips NA. Ageing and bilingualism: Absence of a "bilingual advantage" in Stroop interference in a nonimmigrant sample. The Quarterly Journal of Experimental Psychology. 2012; 65(2):35669. doi: 10.1080/17470218.2011.604788

[48] Kalantaree R. [Cultural superiority of the majority language over the minority (Persian)]. Pazhooheshname-ye Amoozeshi. 2010; 119:27-32.
[49] Blom E, Küntay AC, Messer M, Verhagen J, Leseman P. The benefits of being bilingual: Working memory in bilingual Turkish-Dutch children. Journal of Experimental Child Psychology. 2014; 128:10519. doi: 10.1016/j.jecp.2014.06.007

[50] Morales J, Calvo A, Bialystok E. Working memory development in monolingual and bilingual children. Journal of Experimental Child Psychology. 2013; 114(2):187-202. doi: 10.1016/j.jecp.2012.09.002

[51] Bialystok E, Viswanathan M. Components of executive control with advantages for bilingual children in two cultures. Cognition. 2009; 112(3):494-500. doi: 10.1016/j.cognition.2009.06.014

[52] Ghawami H, Raghibi M. Daryadar M. [Impact of English proficiency level on performance in a computerized, English Version of colorWord Interference Test (Persian)]. Paper perented at the $5^{\text {th }}$ Internation Conference of Cognitive Science. 20 July 2016, Tehran, Iran.

[53] Long EC, Hill J, Luna B, Verhulst B, Clark DB. Disruptive behavior disorders and indicators of disinhibition in adolescents: The BRIEF-SR, anti-saccade task, and D-KEFS color word interference test. Journal of Adolescence. 2015; 44:182-90. doi: 10.1016/j.adolescence.2015.07.003

[54] Jones Chesters M. D-KEFS validity: An update of the research. Technical Report. Oxford: Pearson Assessment; 2008.

[55] Pasha Sharifi H. Preliminary study on gardner's theory of multiple intelligences in the classroom and compatibility issues on students. Journal of Educational Innovations. 2005; 4(11):11-34.

[56] Green CS, Bavelier D. Exercising your brain: A review of human brain plasticity and training-induced learning. Psychology and Aging. 2008; 23(4):692-701. doi: 10.1037/a0014345

[57] Poarch GJ, van Hell JG. Executive functions and inhibitory control in multilingual children: Evidence from second-language learners, bilinguals, and trilinguals. Journal of Experimental Child Psychology. 2012; 113(4):535-51. doi: 10.1016/j.jecp.2012.06.013

[58] Poarch GJ, Bialystok E. Bilingualism as a model for multitasking. Developmental Review. 2015; 35:113-24. doi: 10.1016/j.dr.2014.12.003

[59] Gollan TH, Kroll JF. Bilingual lexical access. In: Rapp B, editor. Handbook of cognitive neuropsychology: What deficits reveal about the human mind. New York: Psychology Press; 2001.

[60] Paap KR, Greenberg ZI. There is no coherent evidence for a bilingual advantage in executive processing. Cognitive Psychology. 2013; 66(2):232-58. doi: 10.1016/j.cogpsych.2012.12.002

[61] Mahdavy B. The role of multiple intelligences (MI) in listening proficiency. Paper presented at the $2^{\text {nd }}$ Biennial International Conference on Teaching and Learning of English in Asia: Exploring New Frontiers (TELiA2). 14-16 June 2007, Changlun, Malaysia.

[62] Breckenridge L. Development throught the lifespan. Boston: Allyn \& Bacon; 2006 